Please do not remove this page

RMIT

UNIVERSITY

\title{
Graphenized carbon nanofiber: a novel light-trapping and conductive material to achieve an efficiency breakthrough in silicon solar cells
}

Chen, Xi; Jia, B; Cai, B; Fang, J; Chen, Z; Zhang, X; Zhao, Y

https://researchrepository.rmit.edu.au/esploro/outputs/9921864292101341/filesAndLinks?institution=61RMIT_INST\&index=null

Chen, X., Jia, B., Cai, B., Fang, J., Chen, Z., Zhang, X., Zhao, Y., \& Gu, M. (2015). Graphenized carbon nanofiber: a novel light-trapping and conductive material to achieve an efficiency breakthrough in silicon solar cells. Advanced Materials, 27(5), 849-855. https://doi.org/10.1002/adma.201404123

Document Version: Accepted Manuscript

Published Version: https://doi.org/10.1002/adma.201404123

Repository homepage: https://researchrepository.rmit.edu.au

(c) 2014 WILEY-VCH Verlag GmbH and Co. KGaA, Weinheim

Downloaded On 2023/04/26 22:40:57 +1000 
Thank you for downloading this document from the RMIT Research Repository.

The RMIT Research Repository is an open access database showcasing the research outputs of RMIT University researchers.

RMIT Research Repository: http://researchbank.rmit.edu.au/

\section{Citation:}

Chen, X, Jia, B, Cai, B, Fang, J, Chen, Z, Zhang, X, Zhao, Y and Gu, M 2015, 'Graphenized carbon nanofiber: A novel light-trapping and conductive material to achieve an efficiency breakthrough in silicon solar cells', Advanced Materials, vol. 27, no. 5, pp. 849-855.

See this record in the RMIT Research Repository at:

https://researchbank.rmit.edu.au/view/rmit:34725

Version: Accepted Manuscript

Copyright Statement:

(C) 2014 WILEY-VCH Verlag GmbH and Co. KGaA, Weinheim

\section{Link to Published Version:}

https://dx.doi.org/10.1002/adma.201404123 


\section{Article type: Communication}

\section{Graphenized carbon nanofiber: a novel light-trapping and conductive material to achieve an efficiency breakthrough in silicon solar cells}

Xi Chen, Baohua Jia, Boyuan Cai, Jia Fang, Ze Chen, Xiaodan Zhang, Ying Zhao, and Min $G u^{*}$

Dr. Xi Chen, Dr. Baohua Jia, Boyuan Cai, Prof. Min Gu

Centre for Micro-Photonics, Faculty of Science, Engineering and Technology, Swinburne

University of Technology, Hawthorn, Victoria 3122, Australia

E-mail:mgu@swin.edu.au

Jia Fang, Ze Chen, Prof. Xiaodan Zhang, Prof. Ying Zhao

Institute of Photo Electronics thin Film Devices and Technology, Key Laboratory of

Photoelectronic Thin Film Devices and Technology, Nankai University, Tianjin 300071,

China

Keywords: graphenized carbon nanofiber, solar cell, light trapping, conductive, plasmonic nanoparticle

The large-scale deployment of environmental friendly photovoltaic (PV) technology promises a radical and might be the only viable solution to the world energy crisis, but has been severely hindered by the slow pace of the efficiency advances achieved in the last 15 years. ${ }^{[1]}$ Nanophotonics strategies have emerged recently as an unparalleled conceptual framework to address the fundamental light-trapping issue in solar cells. Tailored nanomaterials including plasmonic nanoparticles, ${ }^{[2,3]}$ plasmonic nanogratings ${ }^{[4]}$ and dielectric nanoparticles ${ }^{[5]}$ have been developed and integrated with PV devices. However, the demonstrated record efficiency of nanophotonics solar cells is still significantly inferior to that of the conventional ones. ${ }^{[1,3,6]}$ The key reason is due to the often contradictory requirements from the optical and electrical considerations for the nanomaterial design, ${ }^{[2]}$ in particular for state-of-the-art solar cells which have been optimized with little margin to further improve. For an example, although large plasmonic nanoparticles introduce stronger light scattering, the embedding of the large nanoparticles into solar cells leads to the high roughness on the cell structures. In this case the electrical transfer among the cell layers is impeded due to some contact loss, and the fill factor 
reduces dramatically. ${ }^{[2]}$ Therfore to add the critical efficiency breakthrough, the development of advanced nanomaterials that have outstanding light-trapping capabilities but with ignorable optical and electrical losses holds the key.

Recently graphene-based solar cells have emerged at the research forefront of PV devices. ${ }^{[7-10]}$ Graphene, a two-dimensional carbon material exhibits exceptional electrical properties. ${ }^{[11]}$ Additionally, wet chemical method has been used to synthesize low-cost and scalable graphene nanosheets (GNSs). ${ }^{[12]}$ Furthermore, the graphene nanomaterials have good chemical stabilities and can be easily compatible with other nanostructures. ${ }^{[13]}$ Due to these exceptional properties, the graphene-based nanomaterials have great potential to achieve efficiency improvements on the state-of-the-art solar cells. Until nowadays scientists have discovered that graphene materials could improve the electrical properties of PV devices. Ultrahigh efficiency organic solar cells ${ }^{[9]}$ and dye-sensitized solar cells ${ }^{[10]}$ have been fabricated based on graphene nanomaterials.

However, in silicon-based solar cells which have been the dominant products on PV market and have the potential to power skyscrapers, the electrical pathway of the performance gains could not work. In this case a silicon p-n junction takes charge of the exiton separation and the silicon layer has an electron mobility that is substantially higher than organics and $\mathrm{TiO}_{2}$. The solar cell performance could not benefit from the electrical properties improvement through the graphene integration. In the meantime the light absorption in the silicon layer is hardly enhanced, due to a lack of light-trapping mechanism from the two-dimensional GNSs. The strong light absorption of the planar graphene nanostructure reduces the useful silicon layer absorption. Moreover, the nanostructure is also unable to strongly scatter light and significantly enhance the optical path length in the silicon layer. Consequently, it is understandable that $0.8 \%$ has been the highest efficiency enhancement of silicon-based solar cells (including both wafer and thin-film solar cells) reported to date, through the graphene integration. ${ }^{[8]}$ The poor light-trapping property and low conductivity of graphene 
nanomaterials thus are the bottleneck issues impeding their PV application to achieve an efficiency breakthrough.

In the current work, we present an entirely new pathway to solve the bottleneck issues and provide a significant performance improvement on silicon-based solar cells with the state-ofthe-art efficiencies. The concept of the novel nanomaterial and solar cell design is illustrated in Figure 1. It is based on a novel synthesis process in which graphitized carbon fibers are used as the synthesis precursor. Through longitudinal wall cutting by wet chemical method, an innovative one-dimensional carbon material - graphenized carbon nanofiber (GCNF) can be achieved (Figure 1a). Compared with two-dimensional GNSs, GCNFs eliminate the lowenergy excitation character ${ }^{[14]}$ and then exhibit the lower absorption and the stronger back scattering at the long wavelength in which the silicon layer is less absorptive, as shown in Figure 1b. Together with the high electrical conductivity, the GCNFs are applied to be integrated into the back reflector of plasmonic silicon thin-film solar cells (Figure 1c). The light scattering and electrical properties of the plasmonic layer can be improved dramatically. As a result, an efficiency breakthrough based on the state-of-the-art PV devices has been demonstrated.

It has been found that chemical reduction of graphene oxide is a low-cost and large-scale method to prepare GNSs. ${ }^{[13]}$ Graphene oxide, an oxygenated graphene molecule, can be prepared readily by chemical oxidation of graphite using a mixture of potassium permanganate and concentrated sulfuric acid (Hummer's method). ${ }^{[12,15]}$ As a cost-effective and industry-friendly procedure, recently the Hummer's method was also utilized to form graphene nanoribbons by the wall cutting of carbon nanotubes. ${ }^{[16,17]}$ Herein, we select another low-cost one-dimensional carbon material - graphitized carbon fiber as a reaction precursor to synthesize the innovative graphene nanomaterials. The fiber, with an average diameter of 250 $\mathrm{nm}$, consists of an inner amorphous core and outer crystalline walls (see Supporting Information Figure S1). Hummer's method was applied to treat the carbon fiber. The alkenes 
on the fiber walls can be oxidized and opened by permanganate in acid. In the meantime the neighborhood alkenes are distorted and prone to the next permanganate attack. ${ }^{[16]}$ Finally, the fibers are opened longitudinally and the outer walls are peeled off layer by layer, affording an innovative nanomaterial - GCNF, as shown in Figure 1a.

We imaged the novel nanostructures by transmission electron microscope (TEM) (Figure 2a). After the Hummer's treatment, the amorphous core remains and the average diameter of the fiber decreases from $250 \mathrm{~nm}$ to $75 \mathrm{~nm}$. Through the high-resolution TEM image (Inset of Figure 2a), a multi-walled nanostructure can be observed on the outer walls. In the Raman spectrum (Figure 2b) a strong G-mode peak appears at approximately $1580 \mathrm{~cm}^{-1}$, arising from the stretching of the C-C bond in the ordered structure. ${ }^{[18]}$

To confirm the longitudinal wall-opening mechanism during the Hummer's reaction, the introduction amount of potassium permanganate was adjusted and it has been observed that the opening degree can be controlled. When the permanganate amount increased by 5 times, the side walls almost completely unravel and the fiber diameter decreases to $50 \mathrm{~nm}$ (see Supporting Information Figure S2). On the other hand, $100 \mathrm{~nm}$ nanofibers with some cracks on the side walls can be formed once the permanganate amount decreased by 2 times (see Supporting Information Figure S3).

A key to successfully realizing light trapping in silicon-based solar cells is an integration of nanomaterials that exhibit low long-wavelength absorption. Since in the solar cells the short-wavelength incident light can be fully absorbed by the silicon layer, the light-trapping materials can only be effective in the long-wavelength range. ${ }^{[1]}$ In such a range the absorption from the integrated materials that cannot contribute to photocurrent is required to be as low as possible, so that through the material integration the light-trapping effect can overcome the absorption loss. ${ }^{[2]}$ Here we show that the GCNF meets the criteria. The absorption spectra of GCNFs and GNSs were measured and shown in Figure 2c. The water suspensions of twodimensional carbon materials - GNSs and graphite flakes (see Supporting Information Figure 
S4) absorb more light in the long wavelength, due to the low-energy excitation. ${ }^{[8,14]}$ However, in the spectrum of the one-dimensional GCNF the characteristics of the low-energy excitation cannot be observed, and a lower absorption can be observed especially in the wavelength range from $550 \mathrm{~nm}$ to $800 \mathrm{~nm}$. The absorption decreases with the wavelength increase, similar to other one-dimensional carbon materials such as graphitized carbon fibers (see Supporting Information Figure S4) and carbon nanotubes. ${ }^{[19]}$

The low-absorption GCNFs were integrated into single-junction amorphous silicon plasmonic solar cells (Figure 1c). In the solar cell an amorphous silicon layer was deposited by plasma-enhanced chemical vapor deposition (PECVD) as the semiconductor absorbing layer. Then an Al-doped $\mathrm{ZnO}$ layer was sputtered at the rear side for back reflection. $200 \mathrm{~nm}$ lumpy-shaped silver nanoparticles with a broadband plasmon response were embedded inside the $\mathrm{ZnO}$ layer by airbrush spraying to scatter light into a large angle. ${ }^{[2]}$ The GCNFs were also sprayed inside the rear $\mathrm{ZnO}$ layer to avoid direct light shadowing loss. The surface coverage of GCNFs can be easily adjusted by changing the deposition amounts.

To demonstrate the performance improvement of solar cells from the GCNF integration, the relationship between the surface coverage and the enhancement in the short-circuit photocurrent density $\left(J_{s c}\right)$ is shown (Figure 3a). It is obvious that within the surface coverages ranging from $5 \%$ to $10 \%$, GCNFs indeed exhibit a higher $J_{s c}$ enhancement than GNSs do. For the $75 \mathrm{~nm}$ GCNFs, the cells present the consistent $J_{s c}$ enhancement. The largest $J_{s c}$ enhancement of $2.5 \%$ can be achieved under the $10 \%$ coverage. Together with the enhanced fill factor, the energy conversion efficiency increases considerably from $10.6 \%$ to $11.0 \%$ (Table 1), making this device one of the highest-performing single-junction silicon thin-film solar cells reported to date. ${ }^{[6,20]}$ Most importantly, the $3.8 \%$ efficiency enhancement from GCNFs is to our knowledge the highest one ever observed in silicon-based solar cells, significantly breaking the current world record from the integration of graphene materials (0.8\%). ${ }^{[8]}$ A coverage increase from $10 \%$ could not increase the $J_{s c}$ enhancement, because 
herein a large amount of nanofibers can be integrated into solar cells. The mass material absorption provides a negative influence on the solar cell performance. ${ }^{[2]}$

It has been found that the performance improvement can be resulted from the external quantum efficiency (EQE) enhancement between $550 \mathrm{~nm}$ and $800 \mathrm{~nm}$, compared with that of the cells integrated by lumpy silver particles only (Figure 3b). This EQE enhancement is further validated by the measurement of solar cell absorption (Figure 3c), which consistently shows an enhancement in such long wavelength range. The results undoubtedly indicate that the GCNFs can significantly enhance the light absorption in the amorphous silicon layer.

In order to investigate the enhancement mechanism, the back reflectors - Al-doped $\mathrm{ZnO}$ layers embedded with lumpy silver nanoparticles (10\% surface coverage) and graphene nanomaterials were fabricated and the optical spectra were measured. Figure 4a demonstrates the total reflectance spectra of the layers with various surface coverages of GCNFs. For the reflector without nanofibers, the total reflectance gradually enhances with the wavelength increase. It can be attributed to the unique structure of the lumpy silver nanoparticles combining both the large core particles and the small surface particles. Multiple plasmon resonance modes in a very wide wavelength range exist and contribute to broadband light scattering around $600 \mathrm{~nm} \cdot{ }^{[2]}$ With the integration of GCNFs reflectance dips around $380 \mathrm{~nm}$ can be observed, because of the characteristic sp2 absorption peak from the carbon layer. ${ }^{[21]}$ However, the total reflectance decrease cannot degrade the solar cell performances, since in such wavelength the incident light is fully absorbed by the silicon layer. In the long wavelength in which amorphous silicon is less absorptive, a dramatically higher total reflectance is exhibited through the integration of GCNFs under $10 \%$ coverage. We suggest that the surface plasmon resonance of the silver nanoparticles can be optimized in longer wavelength and enhance the back reflection in the required wavelength range. ${ }^{[22]}$ Additionally, when the nanofiber coverage was adjusted to $15 \%$, the long-wavelength total reflectance decreases due to the relatively large nanofiber absorption loss. In contrast, once GNSs were 
integrated into the dielectric layer with lumpy silver nanoparticles, no significant enhancement can be found above $550 \mathrm{~nm}$ (Figure 4b).

A superior light-trapping property requires PV back reflectors exhibiting both high total reflectance and high diffuse reflectance. The diffuse reflectance spectra of the Ag/dielectric layer with GCNFs and GNSs were measured and shown in Figure 4c. The Ag/dielectric layer shows a diffuse reflectance peak in $420 \mathrm{~nm}$ from the plasmon scattering of the lumpy nanoparticles. Once either GCNFs or GNSs were integrated, it is clear that the peak of the diffuse reflectance is red-shifted. Compared with GNSs, the GCNF structure gives a significant enhancement of the diffuse reflectance in the wavelength above $550 \mathrm{~nm}$. In the wavelength range from $600 \mathrm{~nm}$ to $800 \mathrm{~nm}$ the reflector shows extremely high diffuse reflectance above $60 \%$, which is 2 times higher than that reported previously. ${ }^{[23]}$ The results imply that for nanofiber integration the benefit from the plasmon back scattering optimization can overcome the absorption loss, due to the low long-wavelength absorption. On the other hand, the absorption loss of GNSs almost compensates the effect of the scattering optimization, so that only a slight enhancement in solar cell performances can be observed.

To further confirm the light-trapping effect of the GCNF, we also fabricated Al-doped ZnO layer embedded by GCNFs and GNSs (without silver nanoparticles) and measured the diffuse reflectance spectra. In the long wavelength above $550 \mathrm{~nm}$, the nanofiber reflectance is 15\% higher than that of the nanosheets (see Supporting Information Figure S5). Due to the bent carbon layer on the surface, GCNFs contribute to the light back scattering. Consequently, the low absorption and the superior back scattering (including plasmon scattering optimization and graphene scattering) from GCNFs solve the light-trapping issue in the PV application of graphene materials, and provide a significant improvement on the absorption of silicon-based solar cells. The field distributions of graphene-enhanced solar cells are shown in Figure $4 \mathrm{~d}$ and e, simulated by the finite difference time domain (FDTD) method (FDTD solutions, Lumerical). The interaction between lumpy silver nanoparticles and GCNFs 
redirects more light back scattered into the amorphous silicon layer than that from GNSs. Compared with the bare solar cell (see Supporting Information Figure S6), both GNSs and GCNFs combined with lumpy silver nanoparticles can enhance the light absorbance in the amorphous silicon layer by introducing enhanced back scattering properties.

The GCNFs also exhibit exceptional electrical properties. In Supporting Information Figure S7 the performance enhancements from the integration of carbon nanotubes (with the same average diameter) and graphitized carbon fibers have been demonstrated. Compared with that from GCNFs, the deposition of $250 \mathrm{~nm}$ graphitized carbon fibers into the rear side of amorphous silicon solar cells degrades the energy conversion efficiency. In this case the thickness of the dielectric layer is much less than the fiber diameter, leading to some electrical losses at the back surface. ${ }^{[2]}$ Carbon nanotubes can only provide a slight efficiency gain under $5 \%$ coverage. The further coverage increase reduces the efficiency significantly due to the loss of both $J_{s c}$ and the fill factor. Compared with the GCNFs enhancing the $J_{s c}$ and the fill factor, we believe the difference is most likely generated from the material electrical conductivities. To verify this, GCNF and carbon nanotube transparent electrodes were deposited on glass substrates by dip coating. As shown in Figure 5, GCNFs result in significantly better conductivities than those of the carbon nanotubes. The sheet resistances of the GCNF and carbon nanotube electrodes are 230 and $900 \Omega / \square$ at a $550 \mathrm{~nm}$ transmittance of 50\%, respectively. At $80 \%$ transmittance the GCNF transparent electrode has a sheet resistance of $930 \Omega / \square$, almost 8 times lower than that of the carbon nanotube electrode (7070 $\Omega / \square)$. The nanotubes with a hollow nanostructure cannot transfer the photogenerated electrons as quickly as the solid GCNFs, resulting in the degradation of solar cell electrical properties.

In summary, a novel one-dimensional material - GCNF was designed and synthesized by wet chemical method. Graphitized carbon fiber was utilized as a reaction precursor and treated by Hummer's method. The side walls of the fibers are opened along a line and peeled off layer by layer to achieve GCNFs with the nanosized diameters. The nanofiber exhibits the 
minimized absorption loss and the high electrical conductivity. Once it can be integrated into silicon solar cells, the plasmon response of the metal nanoparticles is tuned and the longwavelength back scattering of the cells is enhanced significantly. A dramatic efficiency breakthrough in which the enhancement is almost 5 times higher than the current world record has been achieved. Due to the strong light-trapping property and the high electrical conductivity, the low-cost and scalable GCNFs have great potential in a wide range of applications including solar cells, photodetectors, supercapacitors and biosensors.

\section{Experimental Section}

Synthesis of GCNFs: The suspension of GCNFs was synthesized by chemical reduction of graphitized carbon fiber via Hummer's method. ${ }^{[15]}$ First, graphitized carbon fibers and $\mathrm{NaNO}_{3}$ were mixed with concentrated $\mathrm{H}_{2} \mathrm{SO}_{4}$. Under vigorous stirring, the reducing agent - $\mathrm{KMnO}_{4}$ was added to the suspension. Then $\mathrm{H}_{2} \mathrm{O}_{2}$ was added to the mixture at $98{ }^{\circ} \mathrm{C}$. The GCNF oxide suspension was obtained after the purification. Next, the GCNF suspension can be prepared by the chemical reduction of GCNF oxide. $\mathrm{NaBH}_{4}$ was added into the suspension of GCNF oxide and the mixture is heated at $100{ }^{\circ} \mathrm{C}$ for $2 \mathrm{~h}$. After the reaction the product was centrifuged and washed. For comparison, GNSs were prepared by Hummer's method using graphite as the precursor. ${ }^{[8,24]}$

Solar cell fabrication: The GCNFs were integrated into the rear dielectric layer of the singlejunction amorphous silicon solar cells. The boron-doped zinc oxide (BZO) layer was deposited on a glass by metal organic chemical vapor deposition (MOCVD). The solar cell was fabricated by PECVD at a substrate temperature of $210^{\circ} \mathrm{C}$. The structure of the absorbing layer is $12 \mathrm{~nm} \mathrm{p- \mu c-SiO} / 6 \mathrm{~nm} \mathrm{i}-\mathrm{a}-\mathrm{SiO}_{\mathrm{x}} / 300 \mathrm{~nm} \mathrm{i}-\mathrm{a}-\mathrm{Si} / 25 \mathrm{~nm} \mathrm{n}-\mu \mathrm{c}-\mathrm{SiO}_{\mathrm{x}}$. Then an Al-doped ZnO layer was sputtered. Before the nanomaterial integration, the solar cell samples $\left(1 \mathrm{~cm}^{2}\right)$ were subjected to a 5 min exposure to ethanol solution under sonication. Lumpy silver nanoparticles, prepared by wet chemical method according to literature procedures ${ }^{2}$, were 
sprayed from water suspensions onto the dielectric $\mathrm{ZnO}$ layer using Iwata airbrush, with the coverage of $10 \%$. Then the GCNF was sprayed from the suspension using the same technique, and the surface coverage can be controlled by the integration amounts. Finally, another dielectric ZnO layer was deposited on the top of the nanomaterials followed by the sputtering of the silver back contact.

Material and solar cell characterization: GCNFs were characterized by a Tecnai F20 TEM system and an inVia Raman microscope system. A spectrometer (Perkin Elmer, Lambda 1050) was employed to measure the absorption spectra of the nanomaterials, the reflectance and diffuse reflectance of dielectric layers with nanomaterials. The PV performances of the solar cells were characterized under a simulated AM1.5 spectrum (Oriel-Sol 3A-94023) and the external quantum efficiency (EQE) measurements (PV Measurement QEX10). The sheet resistances of GCNF and carbon nanotube films, deposited by dip coating, were measured by JANDEL RM3000 system.

\section{Acknowledgements}

Min Gu acknowledges the support from the Science and Industry Endowment Fund. Min Gu and Baohua Jia acknowledge the support from the Australian Research Council (DP140100849). Jia Fang, Ze Chen, Xiaodan Zhang and Ying Zhao acknowledge the financial support from the National Basic Research Program of China (Grant No. 2011CBA00706, 2011CBA00707), Science and Technology Support Program in Tianjin (12ZCZDGX03600), Major Science and Technology Support Project of Tianjin City (No. 11TXSYGX22100), and Specialized Research Fund for the PhD Program of Higher Education (20120031110039). Dr. Sergey Rubanov is acknowledged for his assistance with TEM measurements.

Received: ((will be filled in by the editorial staff))

Revised: ((will be filled in by the editorial staff)) Published online: ((will be filled in by the editorial staff)) 
[1] M. A. Green, K. Emery, Y. Hishikawa, W. Warta, E. D. Dunlop, Prog. Photovoltaics 2014, 22, 701.

[2] a) H. A. Atwater, A. Polman, Nature Mater. 2010, 9, 205; b) X. Chen, B. H. Jia, J. K. Saha, B. Y. Cai, N. Stockes, Q. Qiao, Y. Q. Wang, Z. R. Shi, M. Gu, Nano Lett. 2012, 12, 2187; c) Y. N. Zhang, X. Chen, Z. Ouyang, H. Y. Lu, B. H. Jia, Z. R. Shi, M. Gu, Opt. Mater. Exp. 2013, 3, 489; d) M. Gu, Z. Ouyang, B. H. Jia, N. Stockes, X. Chen, N. Fahim, X. P. Li, M. J. Ventura, Z. R. Shi, Nanophotonics 2012, 1, 235-248; e) X. Chen, B. H. Jia, J. K. Saha, N. Stockes, Q. Qiao, Y. Q. Wang, Z. R. Shi, M. Gu, Opt. Mater. Exp. 2013, 3, 27; f) Y. N. Zhang, N. Stocks, B. H. Jia, S. H. Fan, M. Gu, Sci. Rep. 2014, 4, doi: 10.1038/srep04939.

[3] B. H. Jia, X. Chen, J. K. Saha, Q. Qiao, Y. Q. Wang, Z. R. Shi, M. Gu, Photon. Res. 2013, 1, 22-27.

[4] a) M. G. Deceglie, V. E. Ferry, A. P. Alivisatos, H. A. Atwater, Nano Lett. 2012, 12, 2894; b) V. E. Ferry, A. Polman, H. A. Atwater, ACS NANO 2011, 5, 10055.

[5] a) J. R. Nagel, M. A. Scarpulla, Prog. Photovoltaics 2013, 21, 319; b) D. H. Wan, H. L. Chen, T. C. Tseng, C. Y. Fang, Y. S. Lai, F. Y. Yeh, Adv. Funct. Mat. 2010, 20, 3064.

[6] S. Benagli, D. Borrello, E. Vallat-Sauvain, J. Meier, U. Kroll, J. Hoetzel, J. Bailat, J. Steinhauser, 24th European Photovoltaic Solar Energy Conference, Hamburg, Jan 2009.

[7] a) J. T. Han, B. H. Jeong, S. H. Seo, K. C. Roh, S. Kim, S. Choi, J. S. Woo, Nature Commun. 2013, 4, doi:10.1038/ncomms3491; b) F. Bonaccorso, Z. Sun, T. A. C. Ferrari, Nature Photon. 2010, 4, 611; c) N. P. Sahoo, Y. Z. Pan, L. Li, S. H. Chan, Adv. Mater. 2012, 24, 4203; d) Z. L. Zhu, J. N. Ma, Z. L. Wang, C. Mu, Z. T. Fan, L. L. Du, Y. Bai, L. Z. Fan, H. Yan, D. L. Phillips, S. H. Yang, J. Am. Chem. Soc. 2014, 136, 3760; e) Y. H. Chen, W. C. Lin, J. Liu, L. M. Dai, Nano Lett. 2014, 14, 1467; f) J. T. Wang, J. M. Ball, E. M. Barea, A. Abate, J. A. Alexander-Webber, J. Huang, M. Saliba, I. Mora-Sero, J. Bisquert, H. J. Snaith, R. J. Nicholas, Nano Lett. 2014, 14, 724. 
[8] X. Chen, B. H. Jia, Y. N. Zhang, M. Gu, Light Sci. Appl. 2013, 2, doi: 10.1038/lsa.2013.48.

[9] a) J. Liu, G. H. Kim, Y. H. Xue, J. Y. Kim, J. B. Baek, M. Durstock, L. M. Dai, Adv. Mater. 2014, 26, 786; b) J. Liu, M. Durstock, L. M. Dai, Energy Environ. Sci. 2014, 7, 1297; c) Y. Gao, H. L. Yip, K. S. Chen, K. M. O’Malley, O. Acton, Y. Sun, G. Ting, H. Z. Chen, A. Jen, Adv. Mater. 2011, 23, 1903; d) J. M. Yun, J. S. Yeo, J. Kim, H. G. Jeong, D. Y. Kim, Y. J. Noh, S. S. Kim, B. C. Ku, S. I. Na, Adv. Mater. 2011, 23, 4923; e) Y.Wang, S. W. Tong, X. F. Xu, Adv. Mater. 2011, 23, 1514.

[10] a) H. B. Yang, G. H. Guai, C. X. Guo, Q. L. Song, S. P. Jiang, Y. L. Wang, W. Zhang, C. M. Li, J. Phys. Chem. C 2011, 115, 12209; b) L. Kavan, J. H. Yum, M. K. Nazeeruddin, M. Grätzel, ACS Nano 2011, 5, 9171; c) L. Kavan, J. H. Yum, M. Grätzel, ACS Nano 2011, 5, 165; d) M. Y. Yen, C. C. Teng, M. C. Hsiao, P. I. Liu, W. P. Chuang, C. M. Ma, C. K. Hsieh, M. C. Tsai, C. H. Tsai, J. Mater. Chem. 2011, 21, 12880.

[11] a) Y. B. Zhang, T. T. Tang, C. Girit, Z. Hao, M. C. Martin, A. Zettl, M. F. Crommie, Y. R. Shen, F. Wang, Nature 2009, 459, 820; b) J. H. Chen, C. Jang, S. D. Xiao, M. Ishigami, M. S. Fuhrer, Nature Nanotechnology 2008, 3, 206.

[12] L. Qiu, X. W. Yang, X. L. Gou, W. R. Yang, Z. F. Ma, G. G. Wallace, D. Li, Chem. Euro. J. 2010, 16, 10653.

[13] V. Chabot, D. Higgins, A. P. Yu, X. C. Xiao, Z. W. Chen, J. J. Zhang, Energy Environ. Sci. 2014, 7, 1564.

[14] R. R. Nair, P. Blake, A. N. Grigorenko, K. S. Novoselov, T. J. Booth, T. Stauber, N. M. R. Peres, A. K. Geim, Science 2008, 320, 1308.

[15] W. S. Hummers, R. E. Offeman, J. Am. Chem. Soc. 1958, 80, 1339.

[16] D. V. Kosynkin, A. L. Higginbotham, A. Sinitskii, J. R. Lomeda, A. Dimiev, B. K. Price, J. M. Tour, Nature 2009, 458, 872. 
[17] a) A. Narita, X. L. Feng, Y. Hernandez, S. A. Jensen, M. Bonn, H. F. Yang, I. A. Verzhbitskiy, C. Casiraghi, M. R. Hansen, Nature Chem. 2014, 6, 126; b) T. H. Vo, M. Shekhirev, D. A. Kunkel, M. D. Morton, E. Berglund, L. M. Kong, P. M. Wilson, P. A. Dowben, A. Enders, A. Sinitskii, Nature Commun. 2013, 5, doi:10.1038/ncomms4189.

[18] A. C. Ferrari, D. M. Basko, Nature Nanotechnology 2013, 8, 235.

[19] a) E. Gaufres, N. Tang, F. Lapointe, J. Cabana, M. A. Nadon, N. Cottenye, F. Raymond, T. Szkopek, R. Martel, Nature Photon. 2014, 8, 72; b) R. Nakanishi, R. Kitaura, J. H. Warner, Y. Yamamoto, S. Arai, Y. Miyata, H. Shinohara, Sci. Rep. 2012, 3, doi:10.1038/srep01385.

[20] H. Sai, K. Saito, N. Hozuki, M. Kondo, Appl. Phys. Lett. 2013, 102, 053509.

[21] J. X. Geng, L. J. Liu , S. B. Yang, S. C. Youn, D. W. Kim, J. S. Lee, J. K. Choi, H. T. Jung, J. Phys. Chem. C 2010, 114, 14433.

[22] W. C. Tu, Y. T. Chang, H. P. Wang, C. H.Yang, C. T. Huang, J. H. He, S. C. Lee, Sol. Energy Mater. Sol. Cells 2012, 101, 200.

[23] a) M. J. Mendes, S. Morawiec, F. Simone, F. Priolo, I. Crupi, Nanoscale 2014, 6, 4796; b) R. S. A. Sesuraja, T. L. Temple, D. M. Bagnall, MRS Proceedings 2012, 1391, doi: doi: 10.1557/opl.2012.854 .

[24] a) X. P. Li, Q. M. Zhang, X. Chen, M. Gu, Sci. Rep. 2013, 3, doi: 10.1038/srep02819; b) X. R. Zheng, B. H. Jia, X. Chen, M. Gu, Adv. Mater. 2014, 26, 2699. 
Figure 1. a) Synthesis mechanism of GCNFs. b) Light-trapping mechanism of a GNS and a GCNF under long-wavelength light irradiation. c) Structure of a plasmonic amorphous silicon thin-film solar cell with GCNFs integrated in the back dielectric layer.

a

a Carbon fiber

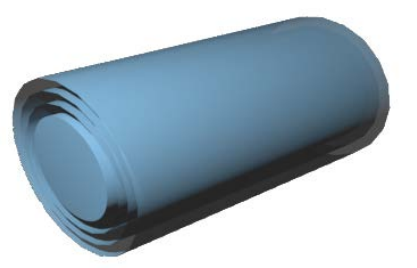

\section{Graphenized carbon} nanofiber
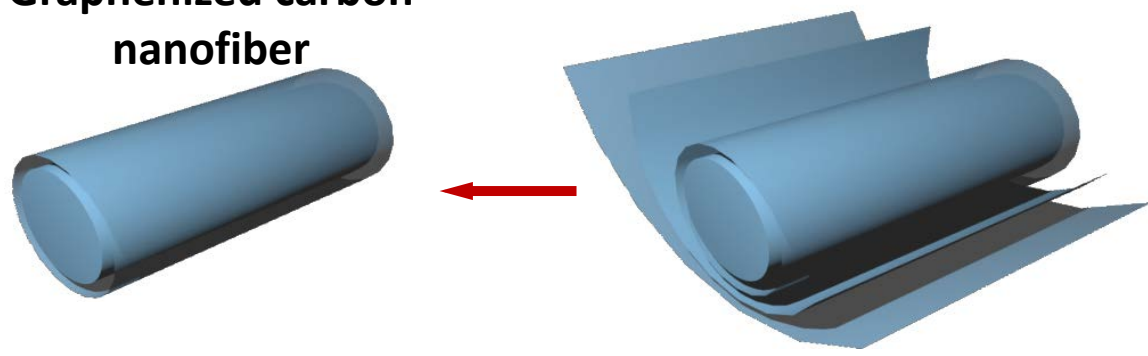

Chemical exfoliation of carbon fiber layers

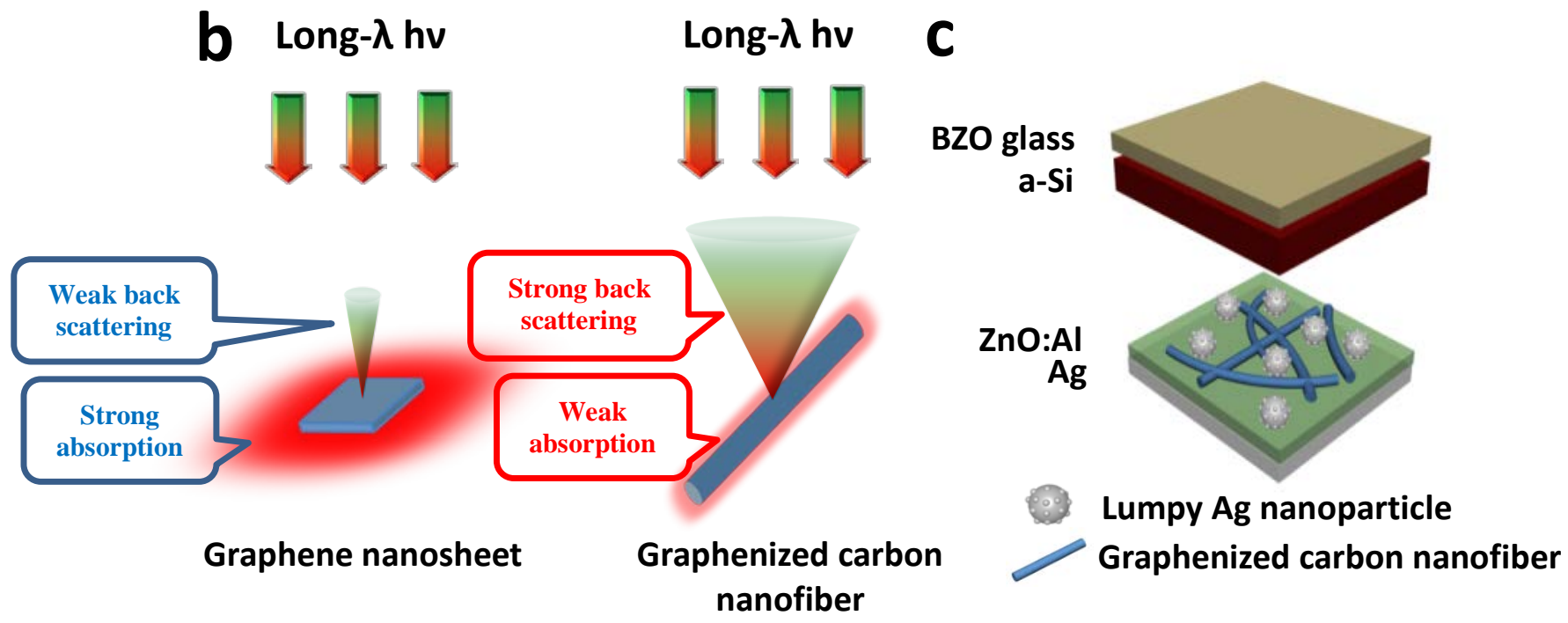


Figure 2. a) TEM image of a GCNF (scale bar: $50 \mathrm{~nm}$ ). Inset: High-resolution TEM image of the outer walls of a GCNF (scale bar: $5 \mathrm{~nm}$ ). b) Raman spectrum of GCNFs. c) UV-vis spectra of aqueous suspensions of GCNFs (red) and GNSs (blue).
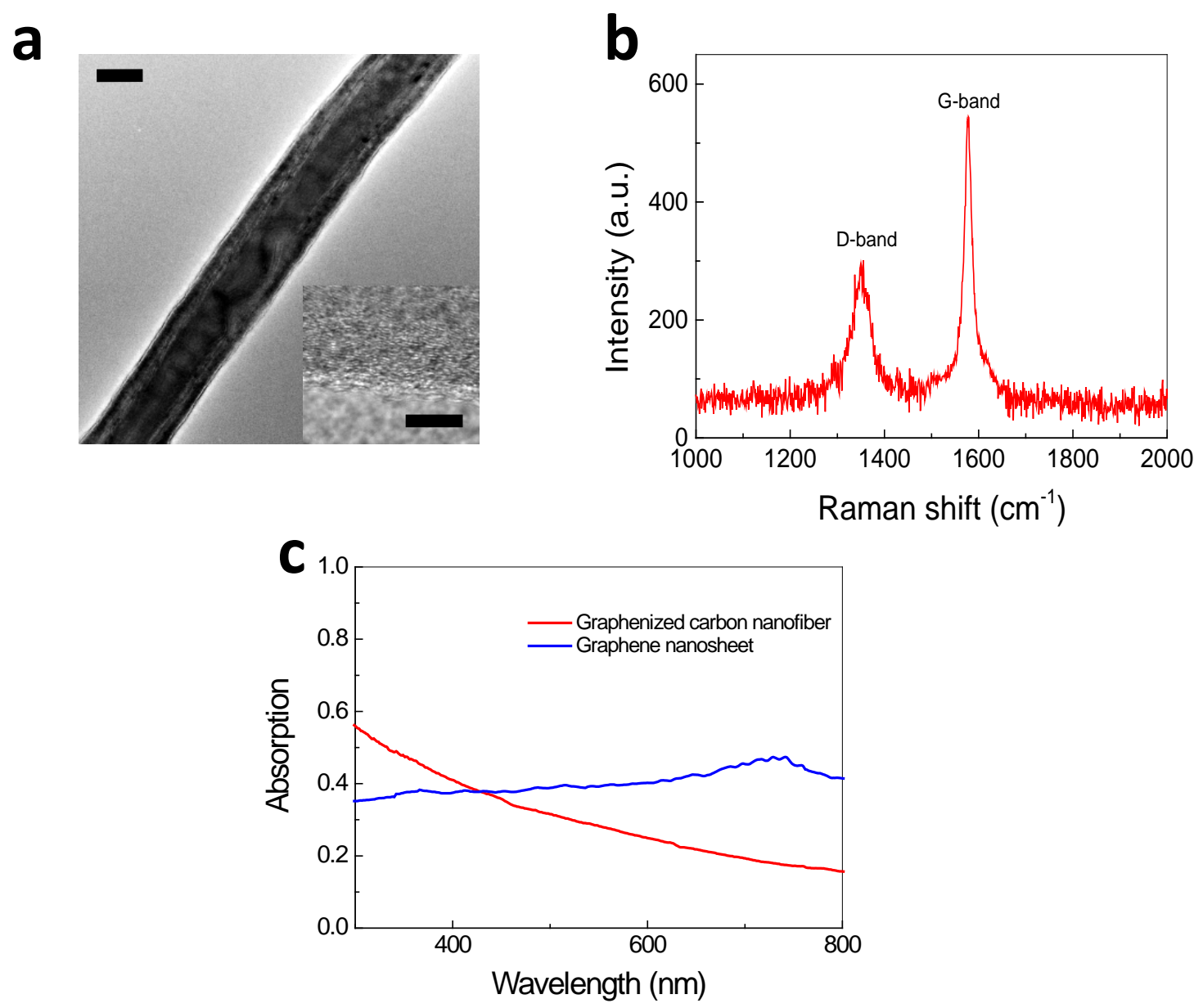
Figure 3. a) Photocurrent enhancement of solar cells with lumpy Ag nanoparticles by the integration of GCNFs (red) and GNSs (blue) under different coverages. b) EQE curves of a-Si cells (black-dash), a-Si cells with silver particles (blue) and a-Si cells with silver particles and $10 \%$ GCNFs (red). c) Absorption enhancement of a-Si solar cells by the integation of lumpy Ag nanoparticles (blue), lumpy Ag nanoparticles and 10\% GCNFs (red).
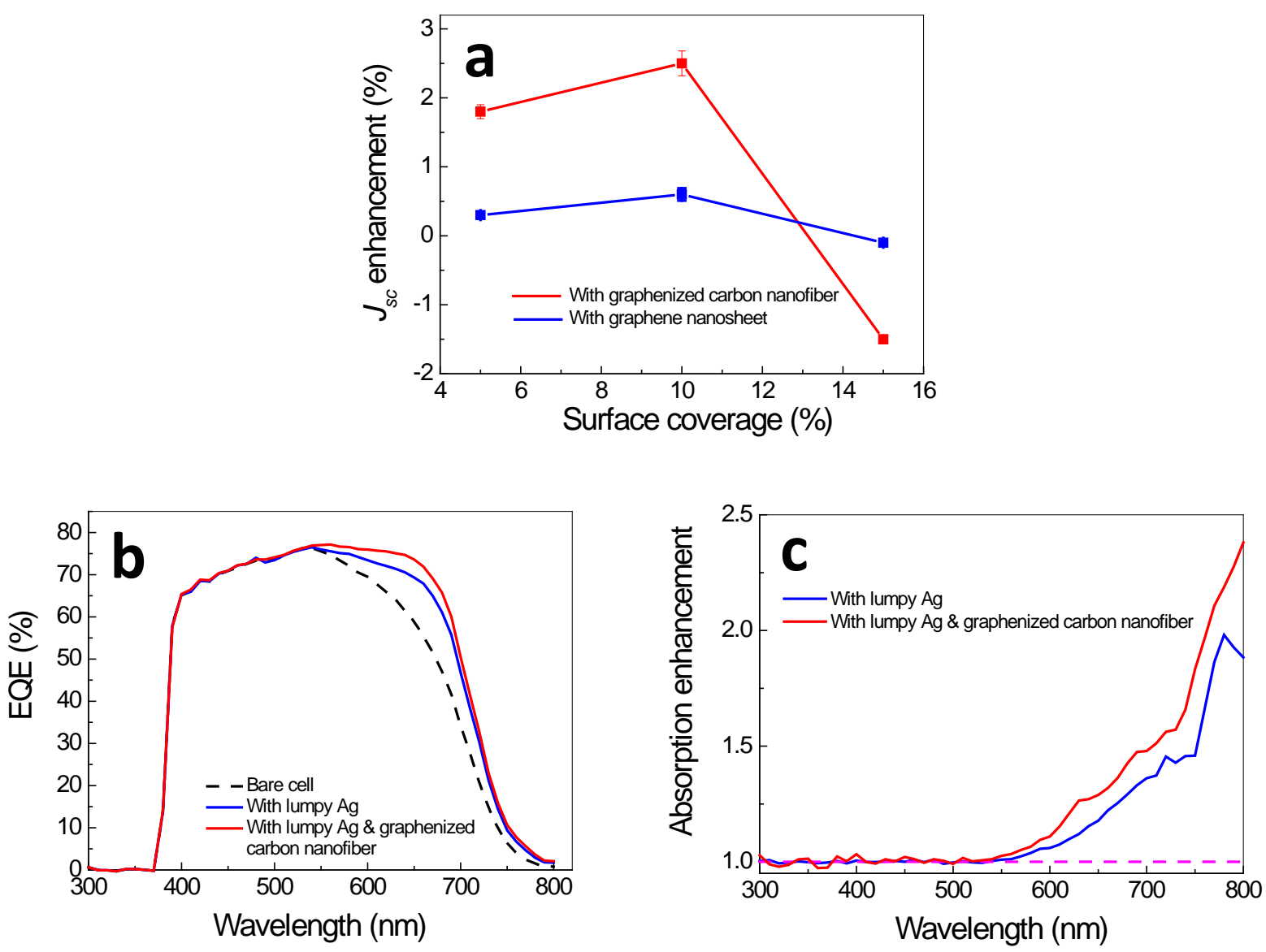
Figure 4. a) Total reflectance of lumpy Ag/dielectric layers with GCNFs under different coverages. b) Total reflectance of lumpy Ag/dielectric layers with GNSs under different coverages. c) Diffuse reflectance of dielectric layers with lumpy Ag (black), lumpy Ag/10\% GNS (blue) and lumpy Ag/10\% GCNF (red). Note: light-trapping effect can only work above the wavelength of $550 \mathrm{~nm}$, indicated by the pink-dash line. d) Field distributions of a-Si cells with lumpy Ag particles and 10\% GNSs at the wavelength of $700 \mathrm{~nm}$. e) Field distributions of a-Si cells with lumpy Ag particles and 10\% GCNFs at the wavelength of $700 \mathrm{~nm}$.
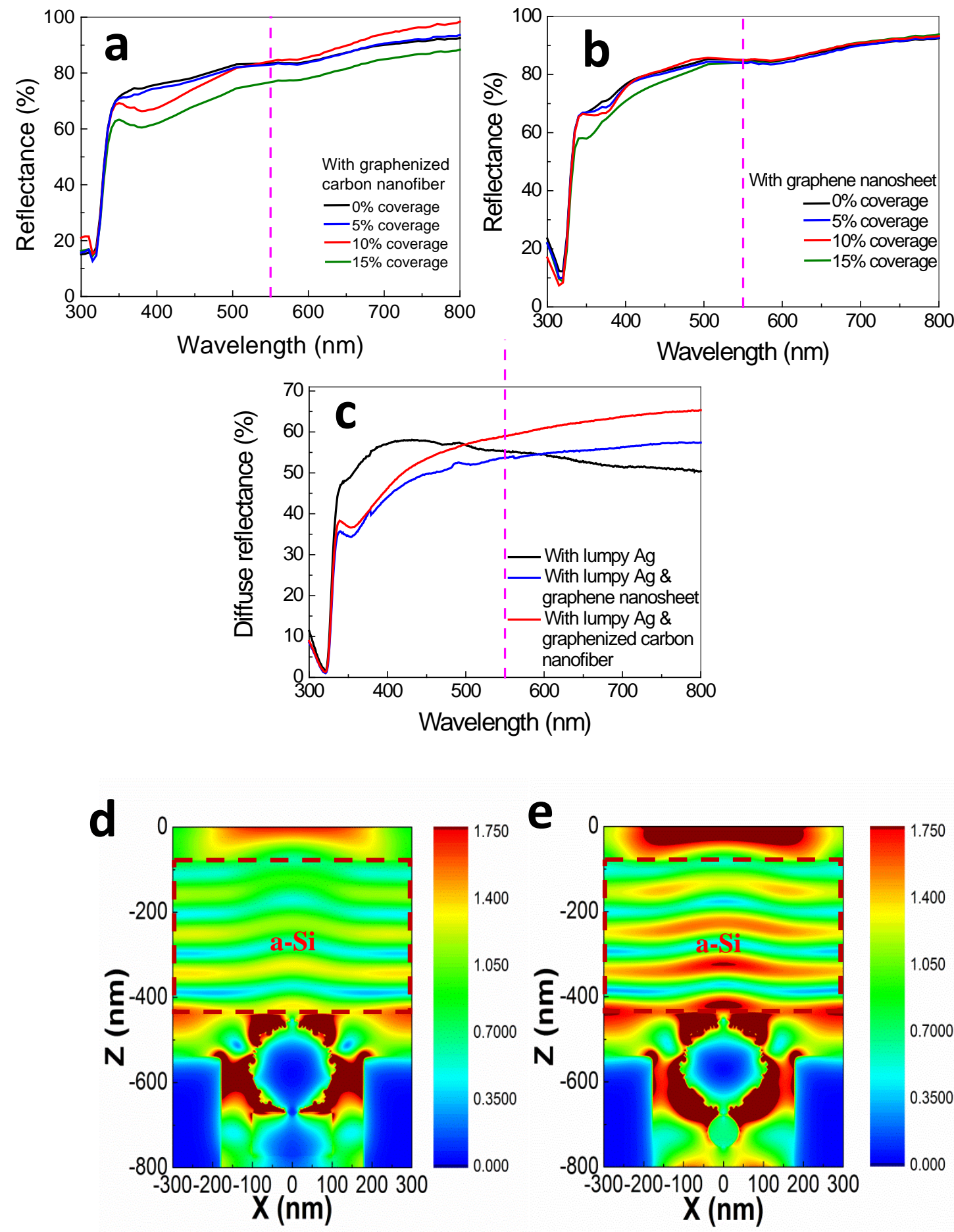
Figure 5. Characteristic sheet resistance-transmittance curves for GCNF (red) and carbon nanotube (blue) transparent electrodes.

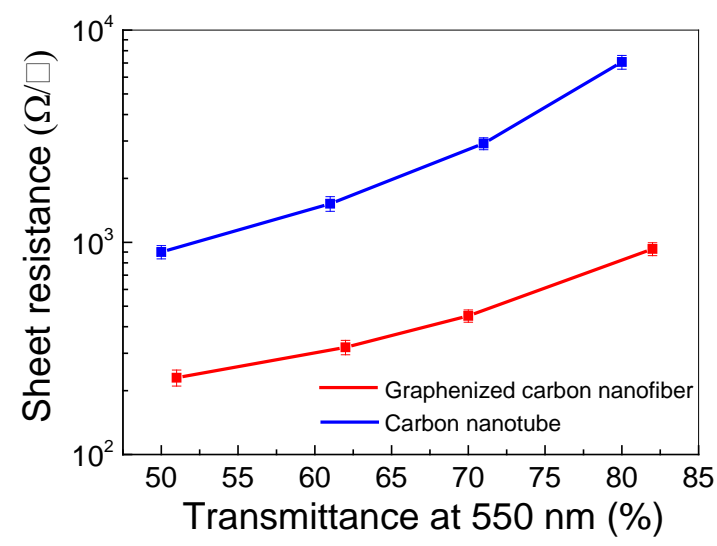


Table 1. Solar cell characteristics for amorphous silicon solar cells, solar cells integrated with lumpy Ag particles, and solar cells integrated with lumpy Ag particles and GCNFs (10\% coverage)

\begin{tabular}{lcccc}
\hline & $\mathrm{J}_{\mathrm{sc}}\left(\mathrm{mA} / \mathrm{cm}^{2}\right)$ & $\mathrm{V}_{\mathrm{oc}}(\mathrm{V})$ & Fill factor & Efficiency $(\%)^{\mathrm{a})}$ \\
\hline a-Si cell & 13.9 & 0.91 & 0.74 & 9.5 \\
$\begin{array}{l}\text { a-Si cell with } \\
\text { lumpy Ag }\end{array}$ & 15.5 & 0.92 & 0.74 & 10.6 \\
$\begin{array}{l}\text { a-Si cell with } \\
\text { lumpy Ag/GCNF }\end{array}$ & 15.9 & 0.92 & 0.75 & 11.0 \\
\hline $\begin{array}{l}\text { a) } 3 \text { samples with similar efficiencies } \\
\text { efficiency enhancements was 5\%. }\end{array}$ & were fabricated. The absolution deviation of the
\end{tabular}


An innovative one-dimensional material - graphenized carbon nanofiber is designed and synthesized. The nanofiber exhibits superior light-scattering property, ultralow absorption loss and high electrical conductivity, and enables a wide range of applications. Simply integrating the nanofibers with the state-of-the-art silicon solar cells leads to a leaping efficiency boost of 3.8\%, almost 5 times higher than the current world record.

\section{Keyword}

graphenized carbon nanofiber, solar cell, light trapping, conductive, plasmonic nanoparticle

Graphenized carbon nanofiber: a novel light-trapping and conductive material to achieve an efficiency breakthrough in silicon solar cells

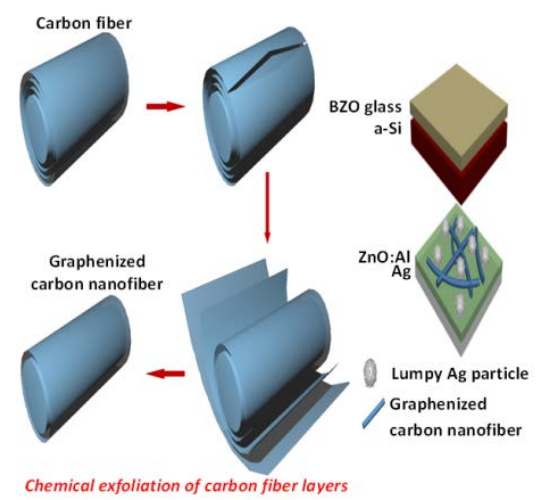

